\title{
Role of the Channel Geometry on the Bubble Pinch-Off in Flow-Focusing Devices
}

\author{
Benjamin Dollet, ${ }^{1, *}$ Wim van Hoeve, ${ }^{1}$ Jan-Paul Raven, ${ }^{2}$ Philippe Marmottant, ${ }^{2}$ and Michel Versluis ${ }^{1}$ \\ ${ }^{1}$ Physics of Fluids, University of Twente, PO Box 217, 7500AE Enschede, The Netherlands \\ ${ }^{2}$ Laboratoire de Spectrométrie Physique, UMR 5588 CNRS, Université Joseph Fourier Grenoble 1, \\ BP 87, 38402 Saint-Martin-d'Hères Cedex, France \\ (Received 4 July 2007; published 25 January 2008)
}

\begin{abstract}
The formation of bubbles by flow focusing of a gas and a liquid in a rectangular channel is shown to depend strongly on the channel aspect ratio. Bubble breakup consists in a slow linear 2D collapse of the gas thread, ending in a fast 3D pinch-off. The 2D collapse is predicted to be stable against perturbations of the gas-liquid interface, whereas the 3D pinch-off is unstable, causing bubble polydispersity. During 3D pinch-off, a scaling $w_{m} \sim \tau^{1 / 3}$ between the neck width $w_{m}$ and the time $\tau$ before breakup indicates that breakup is driven by the inertia of both gas and liquid, not by capillarity.
\end{abstract}

PACS numbers: 47.55.D-, 47.20.Ma, 47.55.db

The production of monodisperse microbubbles is a crucial issue in microfluidics [1], driven by applications in food processing, pharmaceutical sciences, and medicine, for example, for targeted drug delivery with ultrasound contrast agent microbubbles [2]. Flow-focusing techniques have proven to be powerful and versatile tools to achieve monodisperse drops and bubbles. Their working principle is based on a coflow of an internal gas phase and an external liquid phase through a constriction, where the gas is pinched off by the coflowing liquid to release bubbles. Various groups have used flow focusing to produce monodisperse bubbles $[3,4]$ and foams $[5,6]$. In most cases, low-cost soft lithography techniques [7] are used: the produced channels are then rectangular.

Despite the wide use of flow focusing, the precise influence of the channel geometry on the bubble formation process remains unexplored, even though it ultimately determines the bubble characteristics: size, polydispersity and formation frequency. In this Letter, we clarify this issue by characterizing in detail the various stages of the formation process, notably the fast final breakup, for different rectangular channel cross sections, from an elongated rectangle to a square.

Flow-focusing devices consist of two inlet channels, one for the liquid and one for the gas, which converge to a narrow channel followed by an outlet channel [Fig. 1(a)]. We produced the devices by soft lithography techniques: a mold was created from a negative photosensitive material (SU-8 GM1060, Gersteltec SARL) spin coated on a silicon oxide substrate, to imprint [Fig. 1(b)] a reticulable polymer layer (PDMS, Sylgard 184, Dow Corning), which was then bonded to a glass cover plate in a plasma cleaner. Two holes of diameter $1.0 \mathrm{~mm}$ were drilled in the glass, to connect the inlet channels with Teflon tubes of outer diameter $1.06 \mathrm{~mm}$, through which gas and liquid were supplied. Pressurized nitrogen was used, and its overpressure, kept constant at 0.7 bar, was controlled by a pressure regulator (PRG101-25, Omega, regulation accuracy $0.1 \%$ ) connected to a pressure sensor (DPG1000B-30G,
Omega). The liquid used was a $10 \%$ solution of dishwashing liquid (Dreft, Procter \& Gamble) in deionized water, which wets optimally the channel walls [5]. It has a volumetric mass $\rho=10^{3} \mathrm{~kg} / \mathrm{m}^{3}$, a surface tension $\gamma=$ $0.03 \mathrm{~N} / \mathrm{m}$, and a viscosity $\eta=10^{-3} \mathrm{Pas}$. The liquid flow rate was controlled by a syringe pump (PHD 22/ 2000, Harvard Apparatus, flow rate reproducibility $0.05 \%)$. The setup was placed under a microscope (Axiovert $40 \mathrm{CFL}$, Carl Zeiss) with a $40 \times$ objective, which was connected to a high-speed camera (Photron Ultima APX-RS). The camera provides $512 \times 512$ pixel images (field of view $240 \times 240 \mu \mathrm{m}$ ) at 10000 frames per second (fps), to image the bubbles in the outlet channel [Fig. 1(c)], and $32 \times 128$ pixel images (field of view $15 \times 60 \mu \mathrm{m}$ ) at $180000 \mathrm{fps}$ (with an exposure time of $5.6 \mu \mathrm{s}$ ), to resolve the bubble formation and the pinch-off in the channel (Fig. 2). We quantified the collapse leading to breakup by
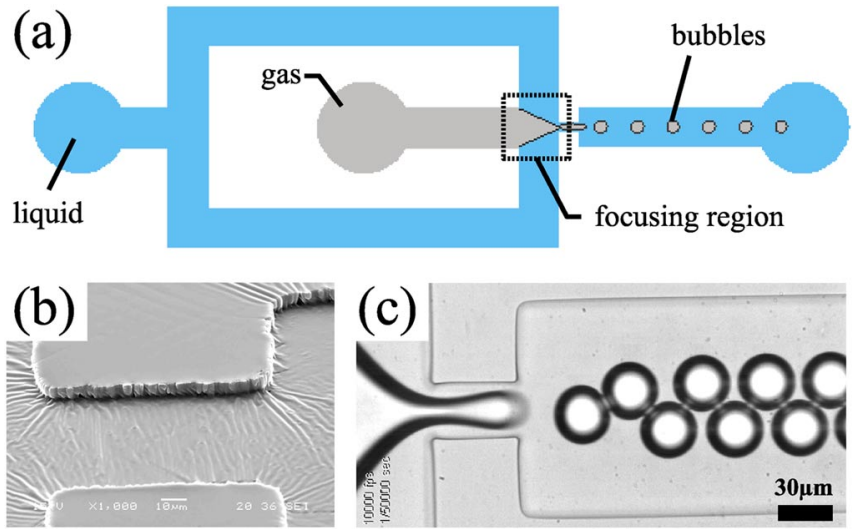

FIG. 1 (color online). (a) Sketch of the flow-focusing setup. (b) Scanning electron micrograph of the imprint in the PDMS slab of the channel of width $30 \mu \mathrm{m}$ and height $6 \mu \mathrm{m}$. (c) Snapshot of the flow-focusing process: the central gas thread is squeezed by the surrounding liquid flow, releasing monodisperse microbubbles, shaped almost as disks because of the confinement in the third dimension. 


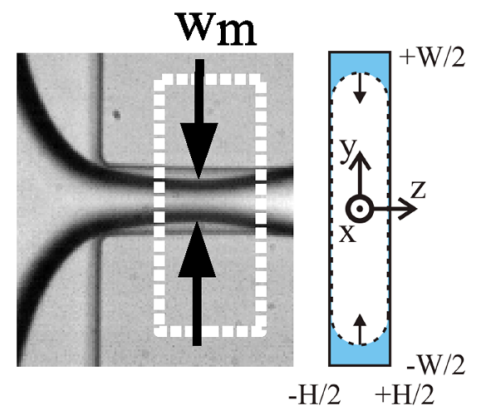

(a) (b)

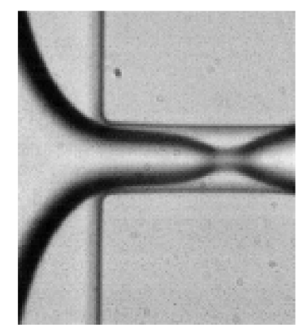

(c)

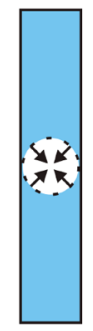

(d)
FIG. 2 (color online). Snapshots of the gas thread in two regimes of collapse described in the text [18]. (a) The gas thread is first squeezed inwards from the sides by the surrounding liquid; $w_{m}$ is its minimal width in the processed region (white dashed rectangle). This regime is sketched in cross section in (b). The gas thread experiences finally a fast pinch-off (c), where it is squeezed radially in the cross section (d).

the minimum width $w_{m}$ of the gas thread [Fig. 2(a)], and we followed its decrease until pinch-off. Actually, the collapse is preceded by a phase during which the gas thread penetrates and fills the channel; we do not study this phase in this Letter. We used three different channels of length $50 \mu \mathrm{m}$, with different aspect ratios for the rectangular cross section: a long rectangle [case a, width $W=$ $30 \mu \mathrm{m}$ and height $H=6 \mu \mathrm{m}$; see Fig. 1(b)], a short rectangle [case $\mathrm{b}$, with a width $(W=30 \mu \mathrm{m})$ comparable to its height $(H=20 \mu \mathrm{m})]$, and a square (case c, $W=$ $H=20 \mu \mathrm{m})$.

We plot for the three geometries the variation of $w_{m}$ during collapse, until breakup, in Fig. 3. We identify two regimes. First, $w_{m}$ decreases linearly with time. We call this regime the $2 \mathrm{D}$ collapse, since $w_{m}$ remains bigger than the channel height, which means that the gas thread is squeezed inwards from the sides [Fig. 2(b)]. The linear decrease has been studied before [4]: at this stage, the gas thread constricts the channel, and the liquid flowing at imposed flow rate $Q_{\ell}$ is forced to squeeze the gas thread at speed $d w_{m} / d t \sim Q_{\ell}$, independently from the gas pressure, the liquid viscosity, and the surface tension. Second, there is a transition from the linear decrease of $w_{m}$ to a fast final pinch-off [Fig. 2(c)]. This happens precisely for $w_{m}=$ $H$ (within less than 5\%); hence, we call this regime the 3D collapse, since the gas thread can be squeezed along any direction [Fig. 2(d)]. Figure 3 also shows that the duration of the 3D collapse is about $20 \mu \mathrm{s}$, with no significant dependence on the cross-section dimensions, contrary to the 2D collapse which, as expected, becomes longer with increasing aspect ratio $W / H$, and is absent for the square channel [Fig. 3(c)].

Garstecki et al. [4] have suggested that the collapse proceeds through a series of equilibria, and justified it on one example, by proving the agreement between experiment and the corresponding computation with surface energy minimization. We now propose a more general stability analysis against perturbations of the gas-liquid interface, suggesting that the 2D collapse is always stable, whereas the 3D collapse is always unstable. To determine qualitatively the influence of the channel confinement on the stability of both $2 \mathrm{D}$ and 3D collapses, we study the linear stability of the gas thread against perturbations of its interface in two simplified channel cross sections: a rectangle with $W \gg H$, and a circular tube, simpler than a square channel and allowing for squeezing of the gas thread along any direction, which is the essential ingredient of the 3D collapse.
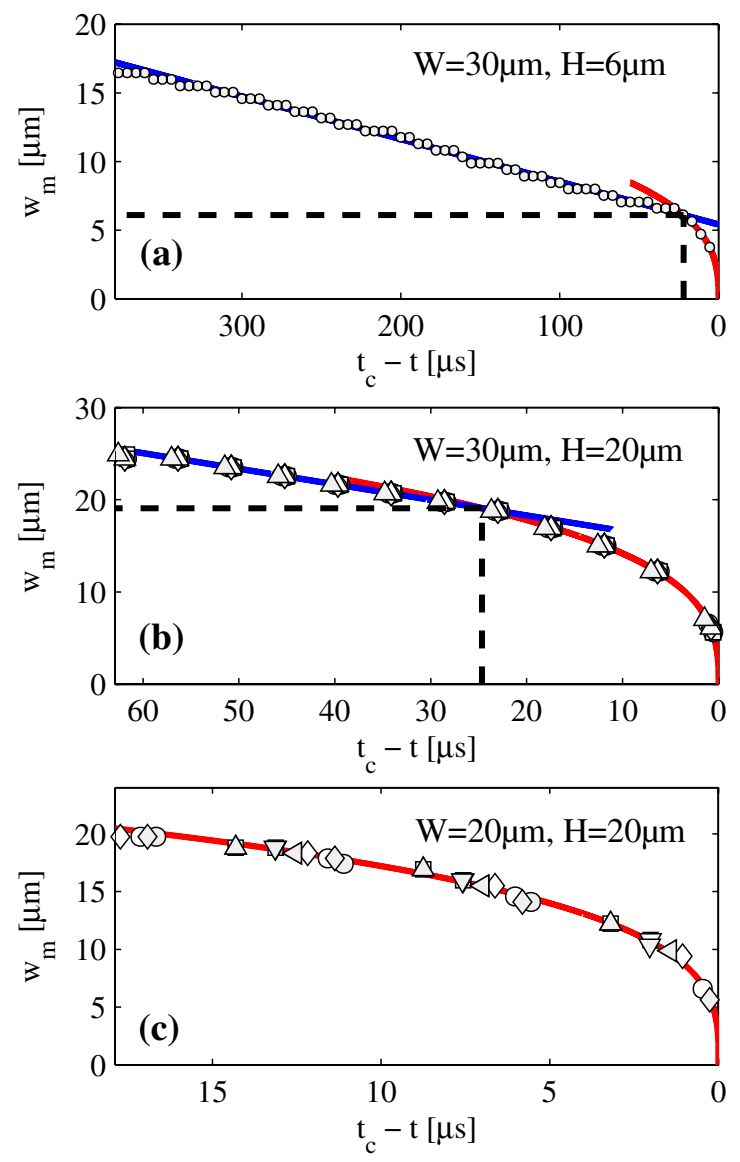

FIG. 3 (color online). Evolution of the minimum width of the gas thread $w_{m}$ as a function of the time $\tau=t_{c}-t$ remaining until final breakup, for the following cross sections $(W \times H$, both in $\mu \mathrm{m}$ ) (a) $30 \times 6$, (b) $30 \times 20$, (c) $20 \times 20$. The liquid flow rates are (a) 20, (b) 20 , and (c) $70 \mu \mathrm{\ell} / \mathrm{min}$. Each curve results from the superposition of independent events (indicated by different symbols in cases $b$ and $c$ ), which collapse on the same master curve, showing the excellent reproducibility of the system. The two regimes described in the text correspond to different fits: first a linear one, and then a power law. Dashed lines in (a) and (b) indicate the transition between the two regimes, where the fitting curves intersect. The transition value between 2D and 3D collapses is $6.1 \mu \mathrm{m}$ in case a $(H=6 \mu \mathrm{m})$, and $19.1 \mu \mathrm{m}$ in case $\mathrm{b}(H=20 \mu \mathrm{m})$. The instant of this transition gives the duration $\tau_{3 \mathrm{D}}$ of the $3 \mathrm{D}$ collapse: $22 \mu \mathrm{s}$ in case a, $25 \mu \mathrm{s}$ in case b, and $19 \mu \mathrm{s}$ in case c. 
In the rectangular geometry, we assume that the cross section of the gas thread is as depicted in Fig. 2(b) a rectangle of dimensions $w_{0} \times H$, bounded with two halfcircles of radius $H / 2$. We thus neglect the thickness of the lubrication films between the gas thread and the channel walls, which is equivalent to assume $\mathrm{Ca}^{2 / 3} \ll 1$ [8] with the capillary number $\mathrm{Ca}=\eta v_{0} / \gamma$. In our experiments, the typical velocity $v_{0}$ is lower than $3 \mathrm{~m} / \mathrm{s}$; thus $\mathrm{Ca}^{2 / 3}<0.2$. We also assume that the fluid flow follows the Hele-Shaw approximation: the spatial variations in the flow along $z$ are much faster than along $x$ and $y$ [see Fig. 2(b) for the definition of the axes], which holds if $W-w_{0} \gg H$. Then the velocity writes $\vec{v}(x, y, z)=\left(1-4 z^{2} / H^{2}\right) \vec{u}(x, y)$, and averaging the Navier-Stokes equation in the gap gives [9] $\rho \frac{\partial \vec{u}}{\partial t}+\frac{6}{5} \rho(\vec{u} \cdot \vec{\nabla}) \vec{u}=-\vec{\nabla} p-\frac{12 \eta}{H^{2}} \vec{u}$. Without the convection term, this equation is the well-known Darcy law. The ratio between the convective and viscous terms gives a Reynolds number $\mathrm{Re} \approx H^{2} u / 10 \nu W$, with $W$ the characteristic length for the spatial variations of the gapaveraged velocity, and $\nu=\eta / \rho$ the kinematic viscosity. We evaluate Re for the dimensions of our elongated rectangular channel: $H=6 \mu \mathrm{m}, W=30 \mu \mathrm{m}$, and since $u<$ $3 \mathrm{~m} / \mathrm{s}$ in our experiments, we compute $\operatorname{Re}<0.4$. We thus use Darcy law in the subsequent analysis.

We assume that the unperturbed flow is at constant velocity $\vec{u}=u_{0} \vec{e}_{x}$, and compute the growth rate of a small perturbation of the interface of the form $w(x, t)=w_{0}+$ $\varepsilon \operatorname{Re}\left(e^{i k x+\sigma t}\right)$, with $k$ real [10]. For this, we express $\vec{u}$ and $p$, using as boundary conditions the continuity of velocity at the interface $\partial w / \partial t=v_{y}-v_{x} \partial w / \partial x$, and the continuity of normal stresses $p=-\gamma \mathcal{C}$ (Laplace pressure), with $\mathcal{C} \simeq$ $2 / H-\partial^{2} w / \partial x^{2}$ the interfacial curvature, assuming $k \varepsilon \ll$ 1 . To close the system, we express mass conservation as $\partial Q_{\ell} / \partial x=2 H \partial w / \partial t$, with the flow rate $Q_{\ell}=$ $2 \int_{-H / 2}^{H / 2} d z \int_{w_{0} / 2}^{W / 2} d y \vec{v}$. The growth rate is then the solution of the dispersion relation:

$$
\left(\sigma+\frac{12 \nu}{H^{2}}\right)\left[\frac{\sigma}{k}\left(1+\xi^{2}\right)-2 i u_{0} \xi^{2}\right]+\frac{2 \gamma k^{2} \xi}{\rho}=0,
$$

where $\xi=\tanh k\left(W-w_{0}\right)$. It is easy to prove from this relation that the real part of the growth rate is always negative; hence, the 2D collapse is always stable.

To study the stability analysis in a 3D, axisymmetric case, we start from a gas thread of constant radius $r_{0}$ in a tube of radius $R$. The present analysis is therefore a special case of the more general stability analysis of core-annular flows [11], where two fluids flow concentrically in a tube. Guillot et al. [12] have recently solved this problem neglecting convection. We adapt their results, neglecting gas viscosity $\eta_{g}\left(\eta_{g} / \eta=0.017\right.$ for nitrogen $)$ : for a perturbation of the gas thread of the form $r(x, t)=r_{0}+$ $\varepsilon \operatorname{Re}\left(e^{i k x+\sigma t}\right)$, we obtain

$$
\begin{aligned}
\sigma= & \frac{\gamma}{16 \eta R a^{5}}\left[-\frac{4 i \nabla P_{0} R^{2}}{\gamma} a^{2}\left(1-a^{2}\right) \tilde{k}\right. \\
& \left.+\left(1-4 a^{2}+3 a^{4}-4 a^{4} \ln a\right)\left(\tilde{k}^{2}-\tilde{k}^{4}\right)\right]
\end{aligned}
$$

with $a=r_{0} / R, \tilde{k}=k r_{0}$, and $\nabla P_{0}=-\partial p / \partial x$ the pressure gradient in the unperturbed case. The growth rate $\operatorname{Re}(\sigma)$ is positive for $0 \leq \operatorname{Re}(\tilde{k}) \leq 1$ and $\operatorname{Im}(\tilde{k})=0$ : contrary to the $2 \mathrm{D}$ case, the $3 \mathrm{D}$ collapse is therefore always unstable. The analysis suggests that the gas thread is destabilized as soon as it can be squeezed radially. This is supported by the fact that the transition between 2D and 3D collapses happens exactly for $w_{m}=H$ [Figs. 3(a) and 3(b)].

We show now that the final breakup is not the result of the growth of a capillary instability. The physical mechanisms driving the $3 \mathrm{D}$ collapse can be inferred from the asymptotic behavior of $w_{m}$ just before breakup [13]. To do so, we now keep only the data in the 3D collapse regime, and plot $\log w_{m}$ versus $\log \tau$ in Fig. 4. All data collapse on power-law master curves, with exponents equal to $0.33 \pm$ 0.03 , compatible with a power law of exponent $1 / 3$. Such an exponent was reported for an asymmetric bubble pinchoff [14], and is related to a nonzero gas flow in the neck. Here, just as in [14], the gas flowing in the neck sucks the surrounding liquid, accelerating the pinch-off compared to the usual bubble pinch-off, where the balance between liquid inertia and surface tension would yield a $1 / 2$ exponent [15]. Hence, although our 3D collapse may initiate from a capillary destabilization of the gas thread, it is eventually driven by gas and liquid inertia. Together with the results of Garstecki et al. [4], who showed experimentally that the $2 \mathrm{D}$ collapse proceeds at a speed independent
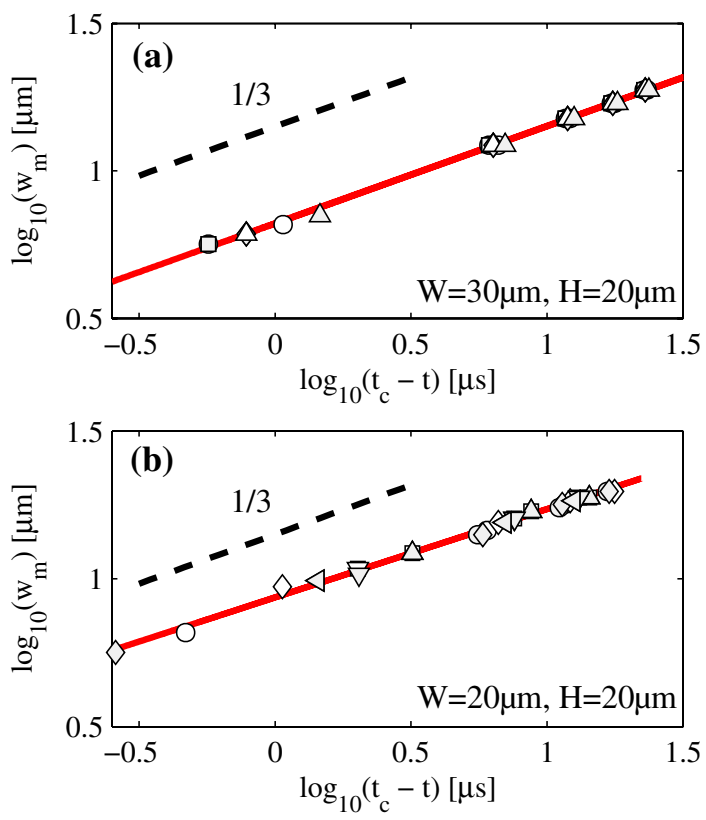

FIG. 4 (color online). Logarithm of the minimum width of the gas thread $w_{m}$ as a function of the logarithm of the time $t_{c}-t$ until final breakup. The line represents the best fit of the data: its slope is (a) 0.33 (cross section $30 \times 20 \mu \mathrm{m}^{2}$ ) and (b) 0.30 (cross section $20 \times 20 \mu \mathrm{m}^{2}$ ). Different symbols represent independent experiments. We do not present the results for the channel of cross section $30 \times 6 \mu \mathrm{m}^{2}$, for which only three data points are in the $3 \mathrm{D}$ collapse regime: in that case, the slope is 0.35 . 
from the surface tension, this shows that the entire collapse does not depend on surface tension, but solely on the inertia of both liquid and gas.

Finally, we measured the polydispersity of the produced bubbles, by imaging them in the outlet channel at a wider field of view. We measured the area of approximately 100 bubbles, each bubble being measured on 50 images to improve the accuracy. We used as the polydispersity index (PDI) the ratio of the standard deviation to the average of the area. Our measured PDI are $0.1 \% \pm 0.1 \%$ (case a), $0.3 \% \pm 0.1 \%$ (case b) and $1.0 \% \pm 0.1 \%$ (case c), confirming that flow focusing is an efficient way to produce very monodisperse bubbles. Moreover, the PDI decreases with increasing aspect ratio of the channel cross section, hence with increasing ratio between the durations of the $2 \mathrm{D}$ and the 3D collapses. Indeed, since the 2D collapse proceeds quasistatically, its reproducibility is limited only by the experimental fluctuations of the gas and the liquid flow rates. On the other hand, the intrinsic unstable nature of the 3D collapse, hence its sensitivity to random fluctuations, limits its reproducibility. More precisely, comparing different pinch-off events in the square and the rectangular $(30 \times$ $20 \mu \mathrm{m}^{2}$ ) channels, we indeed saw a higher standard deviation in pinch-off time (2.8 versus $2.0 \mu \mathrm{s}$ ) and pinch-off location $(1.5$ versus $0.9 \mu \mathrm{m})$ in the square channel.

The values of PDI can be compared to the bubbling frequency. We measured values of 0.18 (case a), 6.9 (case b), and $10.7 \mathrm{kHz}$ (case c). As expected, these frequencies are lower than the inverse time of the process studied in Fig. 3, because the gas tip retracts to the upstream direction after breakup [Fig. 1(c)], and the channel has to be refilled by the gas before the next collapse starts again [5]. This is the bubbling regime, in contrast to the jetting regime where the gas jet would not retract; such a regime has been predicted to happen only for liquid velocities higher than approximately $\gamma / \eta=30 \mathrm{~m} / \mathrm{s}$ [16], much higher than ours ( $3 \mathrm{~m} / \mathrm{s}$ or lower). In the bubbling regime, the durations of the $2 \mathrm{D}$ and $3 \mathrm{D}$ collapses define the upper bound for the bubbling frequency, and at a given channel height, the stable 2D collapse is shortened and eventually suppressed when the aspect ratio $W / H$ decreases to 1 (square channel).

In conclusion, the resolved study of the bubble detachment revealed that the ultimate stage of the pinch-off is controlled only by liquid and gas inertia, which fluctuations cause the jitter in the 3D collapse time. On the other hand, in the 2D collapse, fluctuations are smoothened out by viscosity. Therefore the user has a choice between high monodispersity by using elongated rectangular channels, and high bubbling frequency by using square channels. This study therefore brings new insight in the design of microsystems dedicated to the production of microbubbles of very precise properties, including more complex channel geometries [17].
We acknowledge Professor Detlef Lohse and Professor Howard A. Stone for insightful discussions.

*Present address: Institut de Physique de Rennes, UMR 6251, Université Rennes 1, Bâtiment 11A, 35042 Rennes Cedex, France. benjamin.dollet@univ-rennes1.fr

[1] T. M. Squires and S. R. Quake, Rev. Mod. Phys. 77, 977 (2005); P. Tabeling, Introduction to Microfluidics (Oxford University Press, New York, 2006).

[2] J. R. Lindner, Nat. Rev. Drug Discov. 3, 527 (2004).

[3] C. S. Smith, J. Appl. Phys. 20, 631 (1949); A. M. GañánCalvo and J. M. Gordillo, Phys. Rev. Lett. 87, 274501 (2001); P. Garstecki et al., Appl. Phys. Lett. 85, 2649 (2004).

[4] P. Garstecki, H. A. Stone, and G. M. Whitesides, Phys. Rev. Lett. 94, 164501 (2005).

[5] J. P. Raven, P. Marmottant, and F. Graner, Eur. Phys. J. B 51, 137 (2006).

[6] É. Lorenceau et al., Phys. Fluids 18, 097103 (2006).

[7] Y. N. Xia and G. M. Whitesides, Annu. Rev. Mater. Sci. 28, 153 (1998); D. C. Duffy et al., Anal. Chem. 70, 4974 (1998).

[8] F. P. Bretherton, J. Fluid Mech. 10, 166 (1961).

[9] P. Gondret and M. Rabaud, Phys. Fluids 9, 3267 (1997).

[10] We now show why we can discard the case of a complex $k$. From the form of the perturbation, the pressure writes $p(x, y, t)=f(y) \operatorname{Re}\left(e^{i k x+\sigma t}\right)$. As a consequence of Darcy law, $\Delta p=0$; writing $k=k_{r}+i k_{i}$ and $\sigma=\sigma_{r}+i \sigma_{i}$, one therefore gets $0=\partial^{2} p / \partial x^{2}+\partial^{2} p / \partial y^{2}=\left[f^{\prime \prime}(y)+\left(k_{i}^{2}-\right.\right.$ $\left.\left.k_{r}^{2}\right) f(y)\right] \cos \left(k_{r} x+\sigma_{i} t\right)-2 k_{r} k_{i} \sin \left(k_{r} x+\sigma_{i} t\right)$. A necessary condition for this equality to hold is $k_{i} k_{r}=0$; hence, $k$ is real.

[11] C. E. Hickox, Phys. Fluids 14, 251 (1971); L. Preziosi, K. P. Chen, and D. D. Joseph, J. Fluid Mech. 201, 323 (1989).

[12] P. Guillot et al., Phys. Rev. Lett. 99, 104502 (2007).

[13] J. Eggers, Rev. Mod. Phys. 69, 865 (1997).

[14] J. M. Gordillo et al., Phys. Rev. Lett. 95, 194501 (2005); A. Sevilla, J. M. Gordillo, and C. Martínez-Bazán, J. Fluid Mech. 530, 181 (2005).

[15] M.S. Longuet-Higgins, B. R. Kerman, and K. Lunde, J. Fluid Mech. 230, 365 (1991); H. N. Oğuz and A. Prosperetti, J. Fluid Mech. 257, 111 (1993); J.C. Burton, R. Waldrep, and P. Taborek, Phys. Rev. Lett. 94, 184502 (2005).

[16] A. Sevilla, J. M. Gordillo, and C. Martínez-Bazán, Phys. Fluids 17, 018105 (2005); A. M. Gañán-Calvo, M. A. Herrada, and P. Garstecki, Phys. Rev. Lett. 96, 124504 (2006).

[17] O. Amyot and F. Plouraboué, Phys. Fluids 19, 033101 (2007); K. Hettiarachchi et al., Lab Chip 7, 463 (2007).

[18] See EPAPS Document No. E-PRLTAO-100-059802 for a recording at 50000 frames per second of a single collapse of the gas thread in the orifice of a flow-focusing device. For more information on EPAPS, see http://www.aip.org/ pubservs/epaps.html. 